\title{
Haemorrhagic Transformation of a MELAS Stroke-Like Lesion
}

\author{
Mohamed Reda Bensaidane, Marie-Christine Camden, Martin Savard
}

Keywords: Melas, Mitochondrial disorder, Stroke, Hemorrhage - cerebral

doi:10.1017/cjn.2019.317

Can J Neurol Sci. 2020; 47: 117-118

Mitochondrial encephalomyopathy, lactic acidosis and strokelike episodes (MELAS) is a rare progressive maternally inherited mitochondrial disease that clinically harbours various neurological and systemic manifestations. ${ }^{1}$

We report the case of a 32-year-old female with a previously established diagnosis MELAS (confirmed A-to-G tRNA Leucine (UUR) point mutation at np-3243) who was admitted to the neurology ward in the context of new-onset global aphasia. Cerebral imaging revealed an acute right temporal stroke-like lesion that did not correspond to a specific vascular territory and that was confined to the cortex (Figure 1). Susceptibilityweighted imaging was normal. Head and neck vascular imaging did not reveal any abnormalities. The patient's medications included L-arginine, Co-enzyme Q10, a thromboprophylaxis agent and no antithrombotic medication.

Twelve days after her admission, her neurological condition deteriorated through marked confusion and gait problems. Brain CT revealed a new intraparenchymal haemorrhage (IPH) that occurred within the infarcted zone, suggesting haemorrhagic transformation (Figure 2). The patient died a few days after, being surrounded by family and with appropriate palliative care.
MELAS stroke-like events are characterized by cortical hyperintense diffusion-weighted imaging (DWI) associated with vasogenic oedema. They do not follow a defined arterial territory distribution and have a posterior predilection. ${ }^{2}$ Unlike ischaemic stroke processes, cerebral perfusion has been shown to be preserved in imaging series. ${ }^{3}$ The precise cascade through which cellular damage occurs in stroke-like events is not fully elucidated but appears to be multifactorial. Oxidative phosphorylation failure due to mutation-induced mitochondrial protein translational defect leads to decreased oxygenation of cerebral tissue and eventual neuronal death. ${ }^{4}$ Moreover, neuronal hyperexcitability was observed in the context of a progressive DWI hyperintensity and has been suggested to contribute to increasing cellular energy demands. ${ }^{5}$ Finally, mitochondrial angiopathy has been pathologically demonstrated in MELAS $^{6,7}$ and is thought to contribute to vessel hyperaemia and impaired perfusion in the microvasculature. ${ }^{8}$

Given this distinct pathophysiology, stroke-like events should not be expected to have the same biological behaviour as typical acute ischaemic strokes, where strong disruption of blood-brain barrier and reperfusion injury are the leading mechanisms of haemorrhagic transformation. Whereas gyral laminar necrosis and
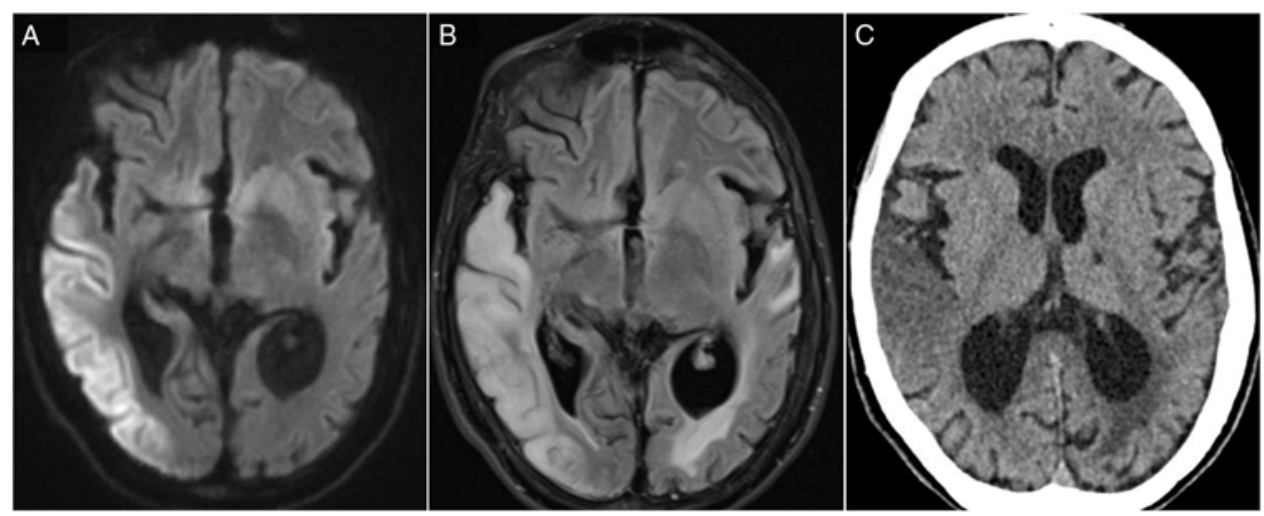

Figure 1: (A) DWI hyperintensity affecting right temporal, parietal and occipital cortices. (B) Corresponding T2-FLAIR hyperintense lesions with additional vasogenic oedema. (C) The same lesion on a CT scan.

From the CHU de Québec, Hôpital de l'Enfant-Jésus, Québec City, QC, Canada (MRB, MCC, MS); Université Laval, Québec, QC, Canada (MRB)

Received June 5, 2019. Final Revisions Submitted September 8, 2019. Date of Acceptance October 18, 2019.

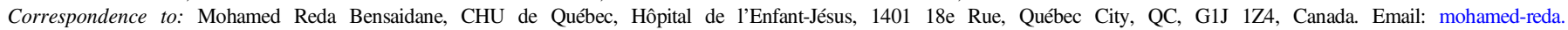
bensaidane.1@ulaval.ca 


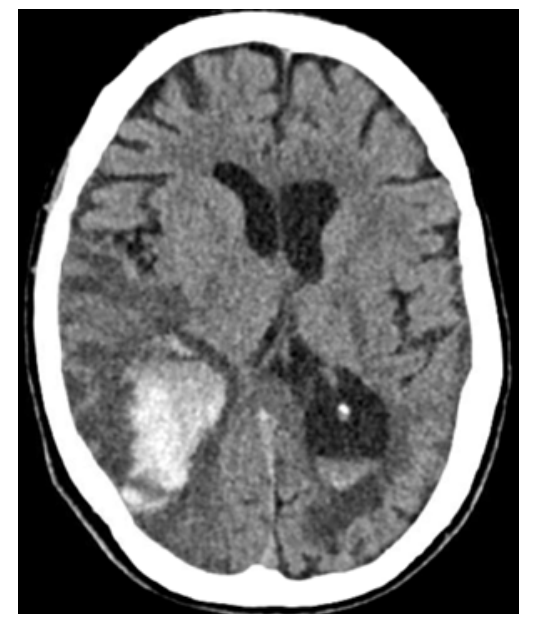

Figure 2: Haemorrhagic transformation within the stroke-like lesion with effacement of the ipsilateral occipital horn of lateral ventricle and intraventricular haemorrhage.

microhaemorrhagic lesions several months after a stroke-like episode have been previously reported, ${ }^{5,9}$ the case reported herein is the first description of haemorrhagic transformation of an acute stroke-like lesion in a MELAS with a m.3243A $>$ G mitochondrial DNA mutation. Furthermore, a case of MELAS with MT-ND5 mutation was reported to display recurrent IPHs, with the last one being fatal. ${ }^{10}$ Altogether, these elements suggest that MELAS stroke-like episodes may have a propensity to cerebral haemorrhages and that haemorrhagic transformation of stroke-like episodes may be underreported.

\section{Statement of Authorship}

$\mathrm{MRB}$ has done the required academic literature review for the conception of the article and drafted the article. MCC and MS have done a critical revision of the article and performed the final approval of the version to be published.

\section{Disclosures}

The authors have no conflicts of interest of declare.

\section{REFERENCES}

1. Testai FD, Gorelick PB. Inherited metabolic disorders and stroke Part 1. Arch Neurol. 2010;67(1):19-24.

2. Yoneda M, Maeda M, Kimura H, Fujii A, Katayama K, Kuriyama M. Vasogenic edema on MELAS: a serial study with diffusionweighted MR imaging. Neurology. 1999;53(9):2182-4.

3. Ito $\mathrm{H}$, Mori $\mathrm{K}$, Harada $\mathrm{M}$, et al. Serial brain imaging analysis of stroke-like episodes in MELAS. Brain Dev. 2008;30(7): 483-8.

4. Sano M, Ishii K, Momose Y, Uchigata M, Senda M. Cerebral metabolism of oxygen and glucose in a patient with MELAS syndrome. Acta Neurol Scand. 1995;92(6):497-502.

5. Iizuka T, Sakai F, Kan S, Suzuki N. Slowly progressive spread of the stroke-like lesions in MELAS. Neurology. 2003;61(9): $1238-44$.

6. Ohama E, Ohara S, Ikuta F, Tanaka K, Nishizawa M, Miyatake T. Mitochondrial angiopathy in cerebral blood vessels of mitochondrial encephalomyopathy. Acta Neuropathol. 1987; 74(3):226-33.

7. Takahashi N, Shimada T, Murakami Y, et al. Vascular involvement in a patient with mitochondrial myopathy, encephalopathy, lactic acidosis, and stroke-like episodes. Am J Med Sci. 2005;329(5):265-6.

8. El-Hattab AW, Adesina AM, Jones J, Scaglia F. MELAS syndrome: clinical manifestations, pathogenesis, and treatment options. Mol Genet Metab. 2015;116(1-2):4-12.

9. Apostolova LG, White M, Moore SA, Davis PH. Deep white matter pathologic features in watershed regions. Arch Neurol. 2005;62(7):1154

10. Pénisson-Besnier I, Reynier P, Asfar P, et al. Recurrent brain hematomas in MELAS associated with an ND5 gene mitochondrial mutation. Neurology. 2000;55(2):317-8. 